\title{
Phasing in and out of the FREEZE-ALL mentality: Was Mother Nature right after all?
}

\author{
David F. Albertini
}

Published online: 15 February 2015

(C) Springer Science+Business Media New York 2015

With all of the twists and turns the field of human ARTs has endured over the years, the one "constant" on the menu has been that of cryopreservation. Call it what you like in technical terms - vitrification, slow-freezing, hypothermic storagebut at the end of the day asking reproductive cells and tissues to take a break from the daily rigors of thermoregulation and progressive aging has become a mainstay for infertility treatment centers. Cryobanking capabilities for most all of the ingredients that could someday be thawed and recombined so as to establish a term pregnancy may have had the elements of an Orwellian fantasy 5 decades ago, but no longer! The arguable prospect being reckoned with today in human ARTs is why not adopt the cryo-road for all ART cycles. Put another way, phasing in the "freeze-all" notion would effectively reduce or eliminate the side effects imposed by controlled ovarian stimulation and the asynchronies inherent in endometrial receptivity and blastocyst implantation potential, issues that have historically conspired in the effective dismemberment of Mother Nature's elegant scheme for human reproduction.

Liquid nitrogen is cheap. Vitrification is now deemed safe and efficient and readily implemented. And reports of live births following years of storage of embryos are increasing in number. Indeed, the reproductive aging clock has stopped. And with it arises the promise of saving your youthful germplasm until the appointed time of warming or thawing and that

Capsule Now that technologies have been optimized to cryopreserve human gametes and embryos - or have they? - the notion of freeze-all IVF cycles has gained momentum and traction. Some of the most pressing issues underlying this change in mentality are discussed with reference to papers in this issue.

D. F. Albertini $(\bowtie)$

University of Kansas Medical Center, Kansas, KS, USA

e-mail: dalbertini@thechr.com final equilibration with a physiological medium that ushers back a metabolically mortal existence.

This month JARG features a series of papers dealing with the topic of freeze-all proposals as broadly applied to human ARTs and more specifically with many issues having to do with vitrification that in some instances reinforce the widespread adoption of this technology in ARTs, but in others raise suspicion as to the short- and long-term consequences of vitrifying reproductively important cells and tissues. Accordingly, we lead with the article of Roque and colleagues that continues to set the stage for discussions into moving from the fresh IVF cycle into one realized after gamete or embryo cryopreservation (Freeze-all policy: is it time? 10.1007/s10815014-0391-0). And to the specific question of how patient age may influence future pregnancy chances when either cleavage stage or blastocysts are subject to vitrification, the group of Fernandez-Shaw offers a firsthand glimpse at a lingering issue that has yet to be fully resolved (Ongoing and cumulative pregnancy rate after cleavage-stage versus blastocyst-stage embryo transfer using vitrification for cryopreservation: impact of age on the results. 10.1007/s10815014-0387-9).

Delving more into the technical details of the vitrification procedure, itself a continued matter of discourse given the lack of an standardized and automated system and clear variability in practice between embryology laboratories, one frequent matter of debate considered "resolved" is the matter of liquid volume when freezing blastocysts. Here the paper by Reed and collaborators takes to task the notion of minimal volume and the implementation of a more robust and reliable method for vitrifying human blastocysts of the biopsied and nonbiopsied varieties (Large-volume vitrification of human biopsied and non-biopsied blastocysts: a simple, robust technique for cryopreservation. 10.1007/s10815-014-0395-9). It is 
noteworthy that embedded within the freeze-all philosophy is not only the tendency to consider development to the blastocyst stage a sign of developmental competence that may forecast pregnancy chances (an opinion not shared by all practitioners), but the "added" prospect that after biopsy and PGS comes a selection and diagnostic advantage not readily obtainable with traditional fresh cycle transfers, especially in patients unable to generate blastocysts.

And finally, there is the matter of where has all the science gone since vitrification and oocyte cryopreservation have been accorded their non-experimental status. Once again, we note the irony in tracing the origins of vitrification as a storage strategy that was first demonstrated for sperm back in a 1949 Nature publication by Professor Polge in the UK. The likes of many an outstanding cryobiologist have since that time guided efforts in the field of ARTs drawing our attention to matters of plasma membrane permeability to water, the notorious and fateful formation of ice-crystals, and re-equilibration at notso-slow and not-so-fast rates, bringing us to what is believed to be state-of-the-art cryogenics for our gametes, embryos, and tissues. In fact, few investigators continue the search for consequences of cryopreservation that could be harmful over the long haul, and this has been especially true since the publication of papers showing little if any effect on the meiotic spindle in human oocytes following vitrification. But there is more to the protein frameworks upon which cells develop, divide, or die.

Enter a component of the cytoskeleton that may be a significant liability when it comes to the impact of vitrification on early human development. Here we speak of the actin cytoskeleton, a dynamic network of filaments that, much as they do in muscle, confer fundamental physical properties on cells that allow them to divide, maintain shape, and position organelles within the cytoplasm. We encourage our readership to consider the paper from Bogliolo and colleagues in Sardinia, taking a novel technological approach in assessing the effects of vitrification in an animal model (Evaluation of the impact of vitrification on the actin cytoskeleton of in vitro matured ovine oocytes by means of Raman microspectroscopy. 10 . 1007/s10815-014-0389-7).

The road to a freeze-all strategy is a timely one to travel. It will clearly open up possibilities for patients and minimize many of the costly and potentially complicating procedures engendered in fresh cycle transfers as we currently know them. As long as we keep open the need to further develop cryostorage approaches and restoring as best we can the properties of gametes and embryos, then the step back to what Mother Nature planned for successful implantation and healthy babies will someday be realized for couples requiring treatment for their infertility. 